\title{
Graphene-based electrochemical supercapacitors
}

\author{
S R C VIVEKCHAND, CHANDRA SEKHAR ROUT, K S SUBRAHMANYAM, \\ A GOVINDARAJ and C N R RAO* \\ Chemistry and Physics of Materials Unit, CSIR-Centre of Excellence in chemistry and DST \\ Unit on Nanoscience, Jawaharlal Nehru Centre for Advanced Scientific Research, Bangalore 560064 \\ e-mail: cnrrao@jncasr.ac.in
}

\begin{abstract}
Graphenes prepared by three different methods have been investigated as electrode materials in electrochemical supercapacitors. The samples prepared by exfoliation of graphitic oxide and by the transformation of nanodiamond exhibit high specific capacitance in aq. $\mathrm{H}_{2} \mathrm{SO}_{4}$, the value reaching up to $117 \mathrm{~F} / \mathrm{g}$. By using an ionic liquid, the operating voltage has been extended to $3.5 \mathrm{~V}$ (instead of $1 \mathrm{~V}$ in the case of aq. $\mathrm{H}_{2} \mathrm{SO}_{4}$ ), the specific capacitance and energy density being $75 \mathrm{~F} / \mathrm{g}$ and $31.9 \mathrm{Wh} \mathrm{kg}^{-1}$ respectively. This value of the energy density is one of the highest values reported to date. The performance characteristics of the graphenes which are directly related to the quality, in terms of the number of layers and the surface area, are superior to that of single-walled and multi-walled carbon nanotubes.
\end{abstract}

Keywords. Graphenes; electrochemical supercapacitors; carbon nanotubes; electrode materials.

\section{Introduction}

Electrochemical supercapacitors are passive and static electrical energy storage devices for applications requiring high power density such as energy back-up systems, consumer portable devices and electrical/ hybrid automobiles. ${ }^{1,2}$ Electrochemical supercapacitors store significantly higher amount of energy than conventional capacitors but less than that of batteries, and are similar in construction to conventional capacitors except that the metal electrodes are replaced by a highly porous electrode. The dielectric film separating the two electrodes is porous and the cell contains a suitable electrolyte such as aqueous (aq.) $\mathrm{H}_{2} \mathrm{SO}_{4}$. Energy is stored in supercapacitors due to the formation of a electrical double layer at the interface of the electrode (electrical double layer capacitors, EDLCs) or due to electron transfer between the electrolyte and the electrode through fast Faradiac redox reactions (psuedocapacitors). In the latter type of supercapacitors, the amount of charge stored is proportional to the voltage. Porous carbon materials such as activated carbon, ${ }^{3,4}$ xerogels, ${ }^{5}$ carbon nanotubes, ${ }^{6-9}$ mesoporous carbon ${ }^{10}$ and carbide-derived carbons $^{11}$ have been investigated for use as electrodes in EDLCs. In the last few years, there has been great interest in graphene, which constitutes an

\footnotetext{
*For correspondence
}

entirely new class of carbon. Electrical characterization of single-layer graphene has been reported. ${ }^{12,13}$ We have investigated the use of graphene as electrode material in electrochemical supercapacitors. For this purpose, we have employed graphene prepared by three independent methods ${ }^{14}$ and compared their supercapacitor behaviour with aq. $\mathrm{H}_{2} \mathrm{SO}_{4}$ as the electrolyte. We have compared the performance of the graphene samples with that of single-walled carbon nanotubes (SWNTs) and multi-walled carbon nanotubes (MWNTs). Furthermore, we have explored the use of ionic liquids as electrolytes. Thus, by employing the ionic liquid, N-butyl-N-methylpyrrolidinium bis(trifluoromethanesulfonyl)imide (PYR ${ }_{14}$ TFSI), we have been able to extend the operating voltage up to $3.5 \mathrm{~V}$ compared to $1 \mathrm{~V}$ normally obtained with aq. $\mathrm{H}_{2} \mathrm{SO}_{4}{ }^{15}$ The present study reveals that graphenes attain a specific capacitance as high as $117 \mathrm{~F} / \mathrm{g}$ in aq. $\mathrm{H}_{2} \mathrm{SO}_{4}$. In the ionic liquid, a specific capacitance $\sim 75 \mathrm{~F} / \mathrm{g}$ is attained with an energy density of $31.9 \mathrm{Wh} \mathrm{kg}^{-1}$.

\section{Experimental}

We have prepared graphene by three methods, which yield samples with different porosities and other characteristics. ${ }^{14}$ The first method involved thermal exfoliation of graphitic oxide. ${ }^{16}$ In this method, graphitic oxide was prepared by reacting 
graphite (Aldrich, $15 \mu \mathrm{m}$ ) with concentrated nitric acid and sulphuric acid with potassium chlorate at room temperature for 5 days. Thermal exfoliation of graphitic oxide was carried out in a long quartz tube at $1050^{\circ} \mathrm{C}$. In the second method, graphene was obtained by heating nanodiamond at $1650^{\circ} \mathrm{C}$ in a helium atmosphere. ${ }^{17}$ The last method involved the decomposition of camphor over nickel nanoparticles. ${ }^{18}$ The reaction was carried out in a two-stage furnace and the camphor was slowly sublimed from the first furnace to the second furnace held at $770^{\circ} \mathrm{C}$ where the micron sized nickel particles were placed. MWNTs were synthesized using the arc discharge process and were purified by heating in air at $700^{\circ} \mathrm{C}$ for one hour. SWNTs were prepared by the arc evaporation of $\mathrm{Ni}-\mathrm{Y}$ catalyst impregnated graphite rods and were purified using hydrogen treatment. ${ }^{19}$

The graphene samples were characterized using transmission electron microscopy (TEM), atomic force microscopy (AFM), X-ray diffraction (XRD) and Raman spectroscopy. TEM images were obtained with a JEOL JEM 3010 instrument fitted with a Gatan CCD camera operating at an accelerating voltage of $300 \mathrm{kV}$. AFM measurements were performed using CP 2 atomic force microscope and Raman spectroscopic characterization was carried out using JobinYvon LabRam HR spectrometer with $632 \mathrm{~nm} \mathrm{HeNe}$ laser. Surface area measurements were carried out in a QuantaChrome Autosorb-1 instrument.
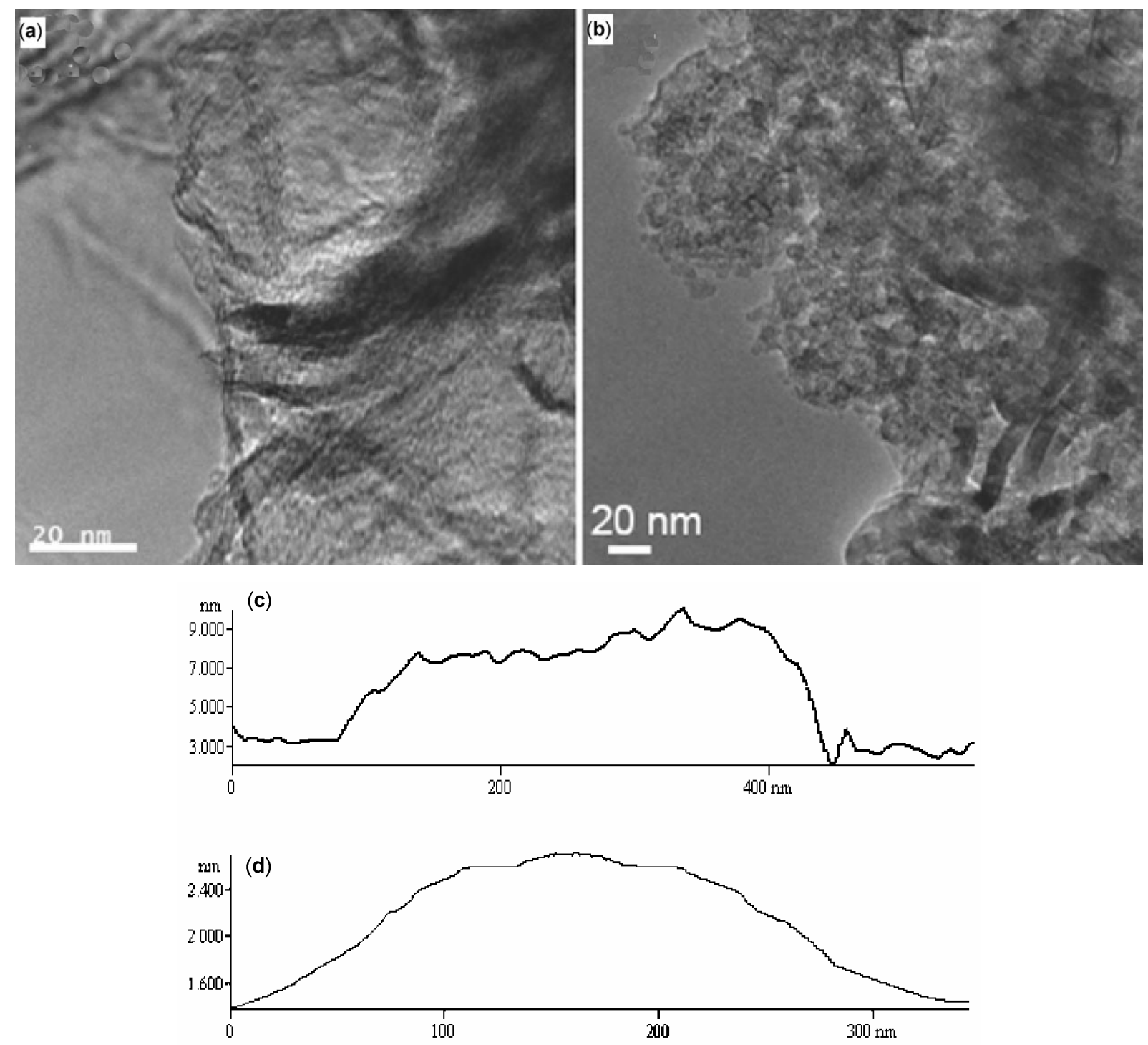

Figure 1. TEM images of (a) graphene obtained by the thermal exfoliation of graphitic oxide (EG). (b) thermal conversion of nanodiamond to graphene (DG). (c) and (d) AFM height profiles of EG and DG respectively. 


\section{Results and discussion}

In figures 1(a) and (b), we show the TEM images of graphene samples prepared by exfoliation of graphitic oxide (EG) and transformation of nanodiamond (DG) respectively. There is some disorder in the graphene sheets of EG while there are nanoparticlelike features in the case of DG. The AFM image profiles of EG and DG show that they consist of 3-6 graphene layers (see figures 1 (c) and (d)). The graphene prepared from camphor (CG) comprises a considerably large number of layers accounting for the sharp graphitic reflections in powder x-ray diffraction pattern, unlike the EG and DG samples. The Brunauer-Emmett-Teller (BET) surface area of EG and DG were 925 and $520 \mathrm{~m}^{2} / \mathrm{g}$ respectively. The

Table 1. Characteristics of the graphene samples.

\begin{tabular}{lcccc}
\hline Sample & $\begin{array}{c}\text { Number } \\
\text { of layers }\end{array}$ & $\begin{array}{c}\text { Crystallite } \\
\text { size }(\mathrm{nm})\end{array}$ & $L_{\mathrm{a}}(\mathrm{nm})$ & $\begin{array}{c}\text { Surface } \\
\text { area }\left(\mathrm{m}^{2} / \mathrm{g}\right)\end{array}$ \\
\hline CG & $>20$ & $6 \cdot 1$ & 10 & 46 \\
EG & $3-6$ & $4 \cdot 7$ & 6 & 925 \\
DG & $3-6$ & $5 \cdot 0$ & 4 & 520 \\
\hline
\end{tabular}
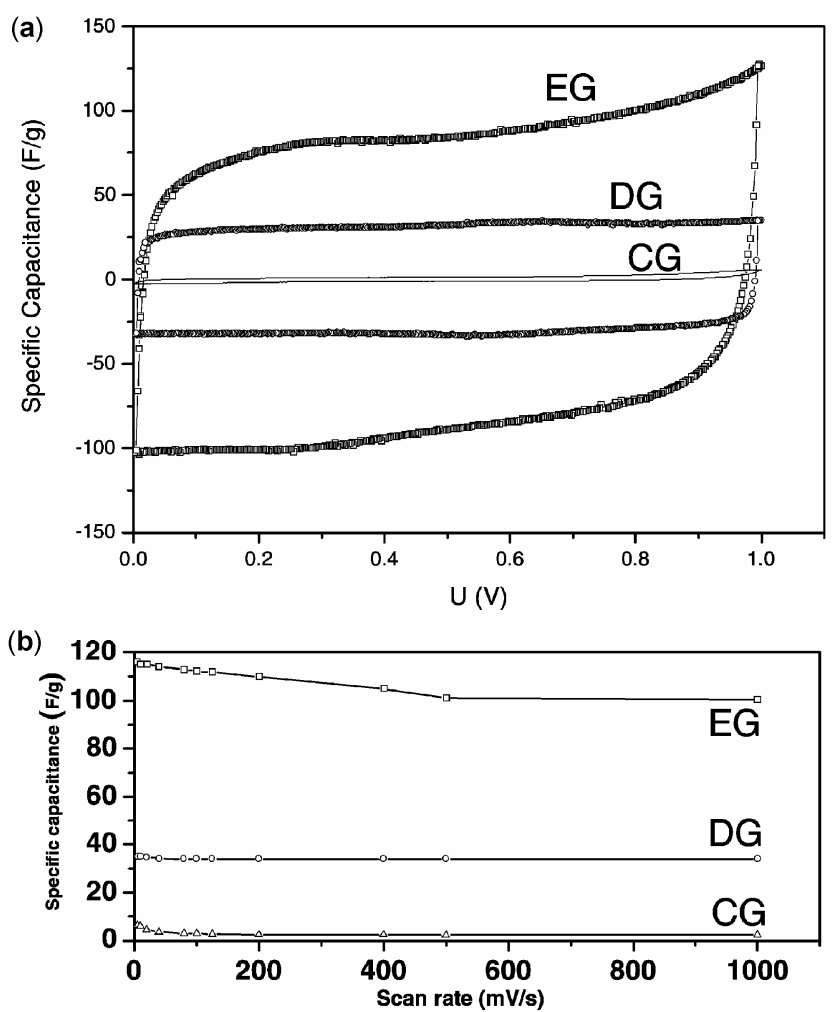

Figure 2. (a) Voltammetry characteristics of a capacitor built from graphene electrodes ( $5 \mathrm{mg}$ each) at a scan rate of $100 \mathrm{mV} / \mathrm{s}$ using (a) aqueous $\mathrm{H}_{2} \mathrm{SO}_{4}(1 \mathrm{M})$ and (b) specific capacitance as a function of scan rate. average pore size was $3 \mathrm{~nm}$ in the case of EG and DG was microporous. CG exhibited type-II adsorption behaviour with a BET surface area of $46 \mathrm{~m}^{2} / \mathrm{g}$. The ratio of the intensities of the Raman $G$ and $2 D$ bands varies in the order $\mathrm{CG}>\mathrm{EG}>\mathrm{DG}$ and the inplane crystallite sizes also varies in the same order (see table 1). From the data in table 1, we conclude that EG and DG have good graphene characteristics.

The graphene electrodes and supercapacitor cells were fabricated following Conway ${ }^{1}$ and the measurements were carried out with a two-electrode configuration, the mass of each electrode being $5 \mathrm{mg}$ in the case of SWNT, MWNT, DG and CG while it was $3 \mathrm{mg}$ for EG. Electrochemical measurements were performed using a PG262A potentiostat/galovanostat, (Technoscience Ltd, Bangalore, India). Experiments with PYR $_{14}$ TFSI-based supercapacitors were carried out at $60^{\circ} \mathrm{C}$ in a mBraun glove box keeping the oxygen and water levels at less than $0 \cdot 1 \mathrm{ppm}$. The ionic liquid was dried at $80^{\circ} \mathrm{C}$ under vacuum for a day prior to the experiment. We have performed cyclic voltammetry as well as constant current charging and discharging to characterize the two-electrode supercapacitor cells with the different graphenes and electrolytes. Specific capacitance was calculated using the following formulae:

In cyclic voltammetry, $C_{\mathrm{CV}}=2\left(i_{+}-i_{-}\right) /(m \times$ scan rate), where $i_{+}$and $i_{-}$are maximum current in the
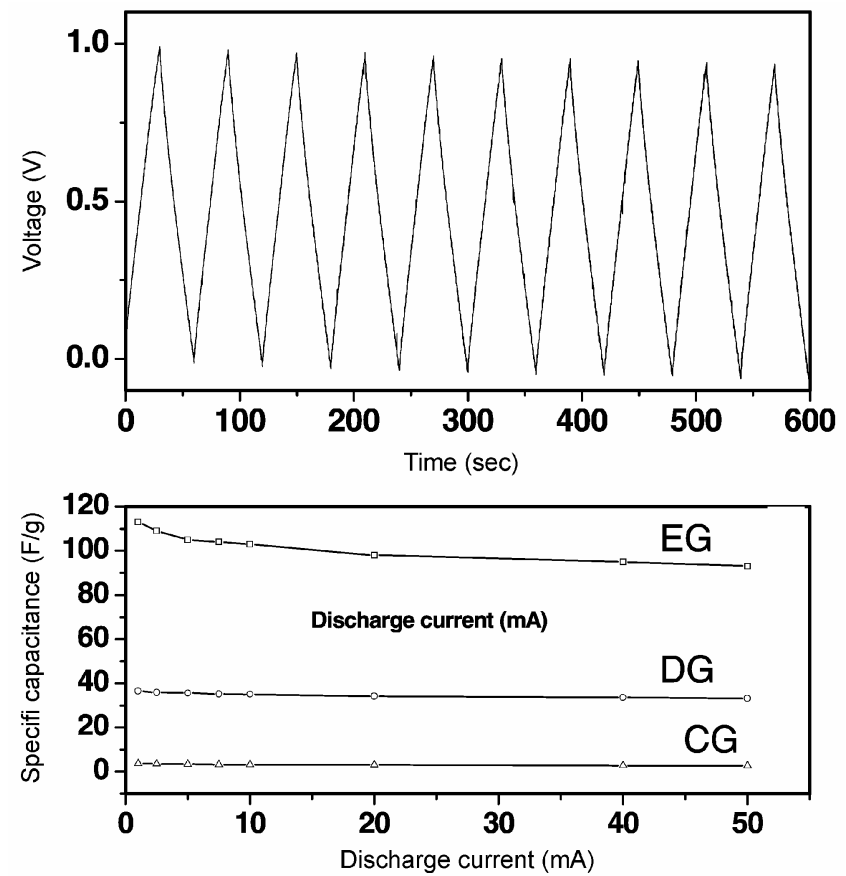

Figure 3. (a) Typical charging and discharging curves of EG at $5 \mathrm{~mA}$ and (b) specific capacitance as a function of discharge current. 
(a)

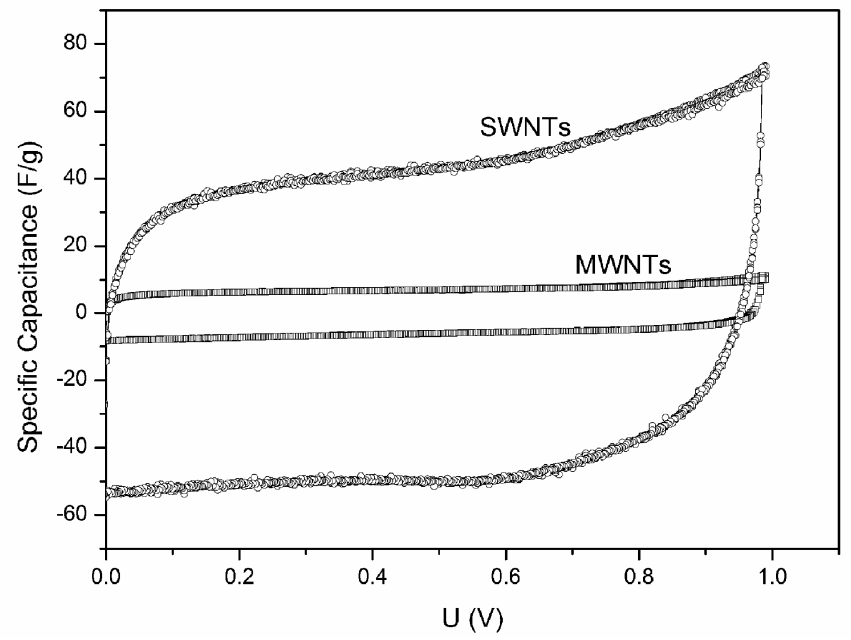

(b) Discharge Current (mA)

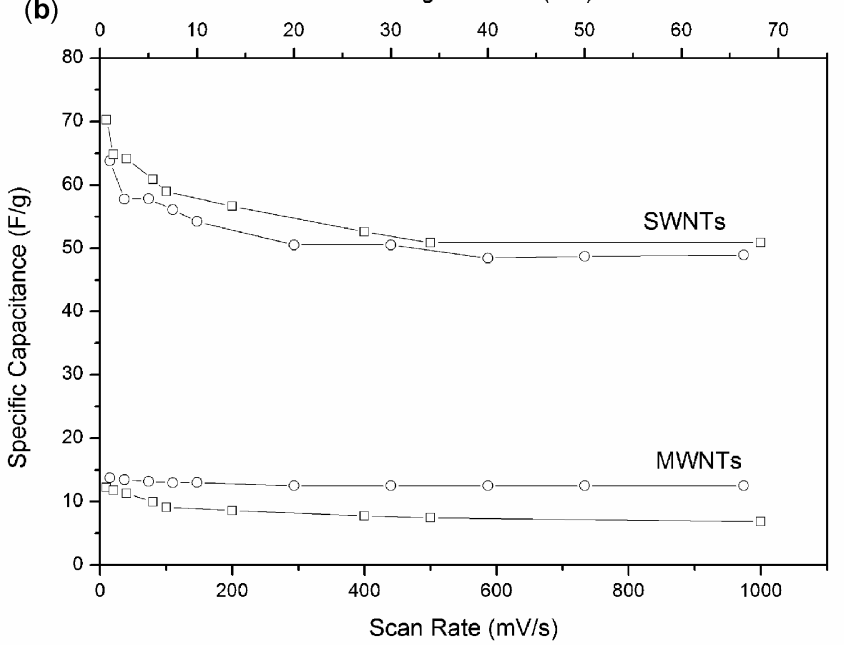

Figure 4. (a) Voltammetry characteristics of a capacitor built from SWNTs and MWNTs at a scan rate of $100 \mathrm{mV} / \mathrm{s}$ using $1 \mathrm{M} \mathrm{H}_{2} \mathrm{SO}_{4}$. (b) Specific capacitance of SWNTs and MWNTs as function of scan rate (open squares) and discharge current (open circles).

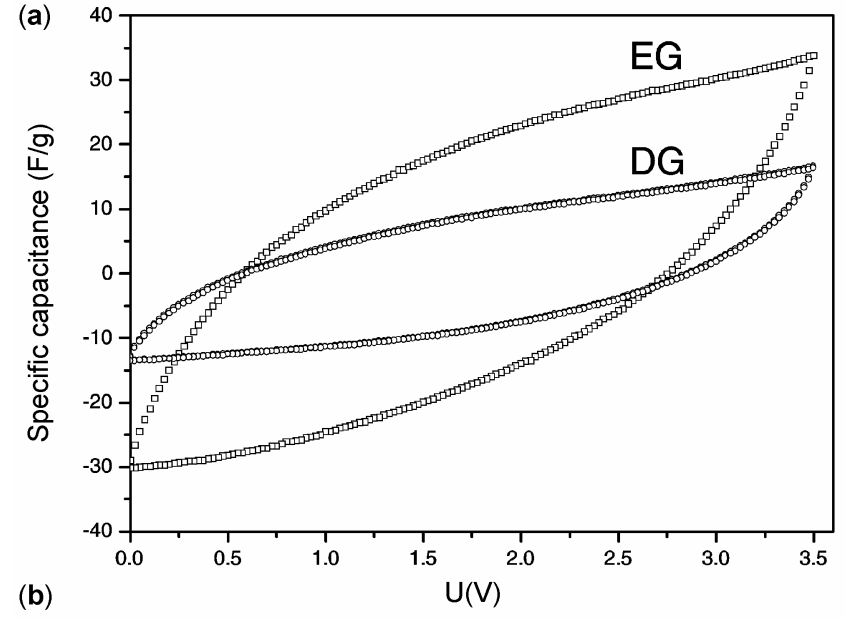

(b)

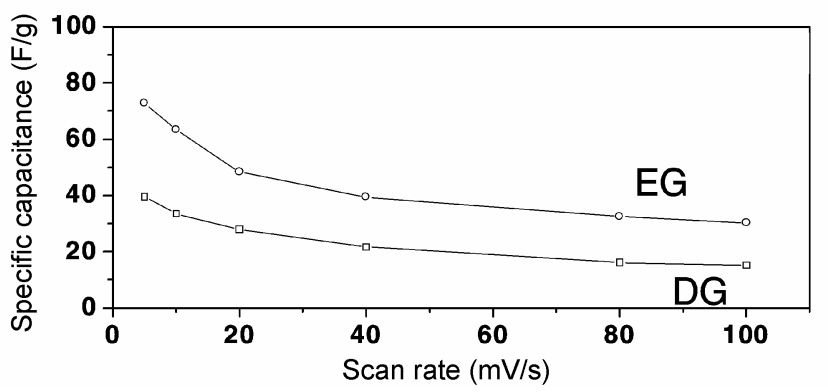

Figure 5. (a) Voltammetry characteristics of a capacitor built from graphene electrodes ( $5 \mathrm{mg}$ each) at a scan rate of $100 \mathrm{mV} / \mathrm{s}$ using ionic liquid. (b) Specific capacitance as a function of scan rate using $\mathrm{PYR}_{14} \mathrm{TFSI}$.

positive scan and negative scan respectively and $\mathrm{m}$ is the mass of electrode.

In constant current charging and discharging, $C_{\mathrm{CD}}=2(i) /(s \times m), i$ is the constant current applied and $s$ is the slope of the discharge curve. The energy density is given as $E=C V^{2}$, where $C$ is the capacitance taking into account both the electrode masses and $\mathrm{V}$ is the operational voltage.

In figure 2(a), we show cyclic voltamograms (CVs) at a scan rate of $100 \mathrm{mV} / \mathrm{s}$ for capacitors built using graphene as electrodes and $1 \mathrm{M} \mathrm{H}_{2} \mathrm{SO}_{4}$ as electrolyte. All the supercapacitors exhibit good box-like CVs with EG exhibiting the highest specific capacitance of $117 \mathrm{~F} / \mathrm{g}$. The specific capacitance decreases slightly as the scan rate is increased as shown in figure 2(b), but the capacitance remains at $100 \mathrm{~F} / \mathrm{g}$ at a scan rate of $1000 \mathrm{mV} / \mathrm{s}$, retaining the box-like characteristics. DG exhibits a capacitance of $35 \mathrm{~F} / \mathrm{g}$ in $1 \mathrm{M} \mathrm{H}_{2} \mathrm{SO}_{4}$ with no change in the specific capacitance even at higher scan rates or discharge currents as seen in figure 2. CG exhibits a low specific capacitance of $6 \mathrm{~F} / \mathrm{g}$.

The results obtained from cyclic voltammetry were confirmed by charge-discharge experiments. In figure 3(a), we show typical charge-discharge curves at $5 \mathrm{~mA}$ current for a supercapacitor built using EG. The charging and discharging curves are linear with no loss due to series resistance. The specific capacitance of EG decreases slightly as the discharge current is increased from $1 \mathrm{~mA}$ to $50 \mathrm{~mA}$, while the other graphene samples exhibited no such dependence (figure 3(b)). There is a significant correlation between the electrochemical results and the AFM or the Raman observations. As the number of layers in the graphene decreases, the value of the surface area 
and specific capacitance increase. The graphene prepared by exfoliation exhibits a capacitance of $12.4 \mu \mathrm{F} / \mathrm{cm}^{2}$ while the graphene prepared by the nanodiamond route exhibits a capacitance of $6.7 \mu \mathrm{F} /$ $\mathrm{cm}^{2}$. The presence of surface functional groups in the EG sample is likely to give rise to some psuedocapacitance.

We have carried out measurements on SWNTs and MWNTs for purpose of comparison. In figure 4(a), we show typical cyclic voltammograms of SWNTs and MWNTs in aq. $\mathrm{H}_{2} \mathrm{SO}_{4}$. The specific capacitance of SWNTs and MWNTs are 64 and 14 F/g respectively and are independent of the scan rate or discharge current as seen in figure 4(b). Clearly, the performance of graphenes is much better than that of carbon nanotubes.

Encouraged by the results obtained using $1 \mathrm{M}$ $\mathrm{H}_{2} \mathrm{SO}_{4}$ as electrolyte we fabricated cells using the ionic liquid $\mathrm{PYR}_{14} \mathrm{TFSI}$ as the electrolyte. We have investigated the EG and DG samples with this electrolyte as they showed promising results in our experiments with aq. $\mathrm{H}_{2} \mathrm{SO}_{4}$. In figure 5 , we show typical $\mathrm{CVs}$ of graphene-PYR ${ }_{14}$ TFSI supercapacitors obtained at a scan rate of $100 \mathrm{mV} / \mathrm{s}$. In $\mathrm{PYR}_{14} \mathrm{TFSI}$, specific capacitance values of $75 \mathrm{~F} / \mathrm{g}$ and $40 \mathrm{~F} / \mathrm{g}$ were obtained with EG and DG respectively. The specific capacitance depends significantly on the scan rate as the ionic liquid is highly viscous (figure $5(b)$ ). The value of the maximum energy density stored using these capacitors are 31.9 and $17.0 \mathrm{Wh} \mathrm{kg}^{-1}$ respectively for EG and DG. These are some of the highest values reported to date and are comparable to those of microporous carbons reported by Balducci et $a{ }^{20}$

\section{Conclusions}

In conclusion, we have shown that graphenes act as good electrode materials for application in supercapacitors. The specific capacitance of the exfoliated graphene in the aqueous electrolyte is comparable to that obtained with activated carbons and superior to that of carbon nanotubes while value of the energy density of the graphene capacitors is one of the highest known to date. The supercapacitor characteristics are directly related to the quality of the graphene specifically the number of layers and the associated surface area. It should be possible to further improve the performance characteristics by modifying or improving the quality of the graphene samples.

\section{References}

1. Conway B E 1999 Electrochemical supercapacitors (New York: Kluwer Academic, Plenum)

2. Kotz R and Carlen M 2000 Electrochim. Acta $\mathbf{4 5}$ 2483

3. Frackowiak E and Beguin F 2001 Carbon 39937

4. Qu D and Shi H 1998 J. Power. Sources 7499

5. Meyer S T, Pekala R W and Kaschmitter J L $1993 \mathrm{~J}$. Electrochem. Soc. 140446

6. Niu C M, Sichel E K, Hoch R, Moy D and Tennent H 1997 Appl. Phys. Lett. 701480

7. Frackowiak E, Metenier K, Bartagna V and Beguin V 2000 Appl. Phys. Lett. 772421

8. An K H, Kim W S, Park Y S, Choi Y C, Lee S M, Chung D, Bae D J, Lim S C and Lee Y $2001 A d v$. Mater. 13497

9. Du C, Yeh J and Pan N 2005 Nanotechnology 16350

10. Yoon S, Lee J, Hyeon T and Oh S M 2000 J. Electrochem. Soc. 1472507

11. Chimola J, Yushin G, Gogotsi Y, Portet C, Simon P and Taberna P L 2006 Science 3131760

12. Novosolev K S, Geim A K, Morozov S V, Jiang D, Zhang Y, Dubonos S V, Grigorieva I V and Firsov A A 2004 Science 306666

13. Geim A K and Novosolev K S 2007 Nat. Mater. 6 183

14. Subrahmanyam K S, Vivekchand S R C, Govindaraj A and Rao C N R 2008 J. Mater. Chem. (in press).

15. Balducci A, Henderson W A, Mastragostino M, Passerini S, Simon P and Soavi F 2005 Electrochim. Acta 502233

16. Schniepp H C, Li J-L, McAllister M J, Sai H, Herrera-Alonso M, Adamson D H, Prud'homme R K, Car R, Saville D A and Aksay I A 2006 J. Phys. Chem. B110 8535

17. Andersson O, Prasad B L V, Sato H, Enoki T, Hishiyama $\mathrm{Y}$, Kaburagi $\mathrm{Y}$, Yoshikawa $\mathrm{M}$ and Bandow $\mathrm{S}$ Phys. Rev. B58 16387

18. Somani P R, Somani S P and Umeno M 2006 Chem. Phys. Lett. 43056

19. Vivekchand S R C, Jayakanth R, Govindaraj A and Rao C N R 2005 Small 1920

20. Balducci A, Dugas R, Taberna P L, Simon P, Plee D, Mastragonstino M and Passerini S 2007 J. Power Sources 165922 